\title{
CDKN3 is an independent prognostic factor and promotes ovarian carcinoma cell proliferation in ovarian cancer
}

\author{
TIANREN LI, HUI XUE, YI GUO and KEJUN GUO \\ Department of Gynecology, The First Hospital of China Medical University, Shenyang 110001, P.R. China
}

Received December 18, 2013; Accepted February 6, 2014

DOI: 10.3892/or.2014.3045

\begin{abstract}
Cyclin-dependent kinase inhibitor 3 (CDKN3) has been reported to promote tumor genesis. Since it is unclear whether CDKN3 participates in the development of epithelial ovarian cancer (EOC), this study assessed the association between CDKN3 expression and cell biological functions, and demonstrated the clinical significance and prognosis of CDKN3 in EOC. CDKN3 expression was evaluated in 97 cases of tumor tissue by immunohistochemistry and in 60 tissues by western blotting. The clinical correlation was analyzed by Kaplan-Meier method and Cox hazards model. The molecular functional roles of CDKN3 in ovarian cancer cell line OVCAR3 were examined by small interfering RNA-mediated depletion of the protein followed by analyses of cell proliferation and invasion. Twenty-three out of $30(76.7 \%)$ human EOC tissues exhibited stronger levels of CDKN3 protein compared with 10 out of 30 (33.3\%) human ovarian surface epithelial (HOSE) tissues. The mean level of CDKN3 expression in the EOC tissues was 3.35-fold that in the HOSE tissues. CDKN3 protein was found to be overexpressed in $68.0 \%$ of the EOC samples and was correlated with poor patient survival $(\mathrm{P}<0.05)$. Furthermore, expression of CDKN3 was significantly associated with FIGO stage, recurrence and residual tumor size $(\mathrm{P}<0.05)$, and the CDKN3 status was a significant prognostic factor for EOC patients $(\mathrm{P}=0.005)$. In addition, depletion of $\mathrm{CDKN} 3$ expression inhibited the growth and clonogenic potential of the OVCAR3 cell line. Our present research found that $\mathrm{CDKN} 3$ may play an important role in the development and proliferation of EOC. CDKN3 may be used as a novel tumor marker to predict the prognosis of EOC.
\end{abstract}

\section{Introduction}

Ovarian cancer is one of three major female malignant tumors of the reproductive system. Its incidence is ranked

Correspondence to: Dr Kejun Guo, Department of Gynecology, The First Hospital of China Medical University, Shenyang 110001, P.R. China

E-mail: guokejun12@gmail.com

Key words: cyclin-dependent kinase inhibitor 3, ovarian cancer, prognosis, proliferation third following cervical and uterine cancer (1). Due to its high malignant potential, the mortality rate of ovarian cancer ranks first among all gynecologic cancers in China. To date, the pathogeny of ovarian cancer is unclear. It is believed that the incidence may be related to environment, hormonal, genetic and other factors. According to different clinical pathological types and genetic features, ovarian cancer can be divided into type I and type II (2). The ovarian malignant progression of type I is low, including low grade serous ovarian cancer, mucinous ovarian cancer and clear cell ovarian cancer. The mutated BRAF or PTEN gene mainly exhibits abnormal expression in this type of ovarian cancer (3). Type II ovarian cancer has a high degree of malignancy, in which BRCA1 and BRCA2 gene mutations are common and the main pathological type is malignant serous ovarian cancer (4-6).

At present, the scientific community believes that a tumor is a disease caused by multiple genomic changes, and tumor progression is based on oncogene activation and/or tumorsuppressor gene deactivation with gene function mutation. Continual growth signal stimulation, evasion of growth inhibition, resistance to the replication of programmed cell death, induction of tumor angiogenesis, and activation of invasion and metastasis are necessary physiological changes leading to different types of malignant tumor cell growth (7). During development, most tumors undergo similar changes in cell physiological characteristics, thus oncogenes and tumor-suppressor genes that are closely associated with these acquired cell behaviors have become a 'hot' topic in the study of tumor molecular mechanisms and cancer treatment.

Cyclin-dependent kinase 3 (CDKN3), a member of the family of protein phosphatase inhibitors, participates in the management of the cell cycle, where it plays a dual role $(8,9)$. CDKN3 plays the role of a cell cycle protein-dependent kinase inhibitor and selectively combines with CDK2 $(10,11)$, reducing $\mathrm{Rb}$ protein (retinal cell tumor protein, a tumorsuppressor gene) phosphorylation (9). Phosphorylation of $\mathrm{Rb}$ protein combined with transcriptional factor E2F1 inhibits cell cycle protein, which is required for G1/S transition, thus blocking G1 to $\mathrm{S}$ phase shift $(12,13)$. CDKN3, also a type of Mdm2 protein, combines with p53 and Mdm2, reduces the sensitivity of p21 and reduces p53 target gene production, so as to promote the progression of the cell cycle (14). The CDKN3 gene was demonstrated to exhibit abnormal expression in many types of tumors (15-17). However, its relationship with ovarian cancer and its molecular role has not been reported. 


\section{Materials and methods}

Patient tissue samples. Ninety-seven ovarian cancer (OC) tissues were collected from patients who underwent routine ovarian resection at The First Affiliated Hospital of China Medical University. None of the specimens were pretreated with preoperative chemotherapy, radiation therapy, or other treatment. All specimens were respectively assessed by two pathologists for pathological diagnosis according to the World Health Organization classification system. The clinicopathologic factors are documented in Table I. Total RNAs and protein were collected from the fresh OC tissues after surgical resection. The study protocol was approved by the Institutional Ethics Committee of China Medical University, and all patients provided written informed consent.

Immunohistochemistry. CDKN3 expression was evaluated by immunohistochemistry (IHC) on 5- $\mu \mathrm{m}$ paraffin-embedded tissue sections. Rabbit anti-CDKN3 (1:200; Santa Cruz Biotechnology, Santa Cruz, CA, USA) was used. Sections were stained with 3,3'-diaminobenzidine. Normal rabbit serum was used as a negative control. The CDKN3 immunostaining score was analyzed according to a semi-quantitative scale. Cytoplasmic/nuclear immunostaining was considered positive staining. The percentage of positive cells was scored as follows: $0(0 \%), 1(1 \%-10 \%), 2(11 \%-50 \%), 3(51 \%-80 \%)$ or $4(>80 \%)$. The intensity of staining was defined as follows: no, '0'; weak, ' '1'; moderate, ' 2 ' and strong staining, ' 3 '. Each sample was given a final score ranging from 0 to 12 . CDKN3 expression was divided into four levels: -, score $0 ; 1+$, score 1-4; moderate expression 2+, score 4-8; and 3+, score 9-12. The immunohistochemical results of CDKN3 were grouped into low expression (0 to $1+$ ) and high expression (2+ to $3+)$.

Western blot analysis. Total protein was separated from tissues and cells using lysis buffer (Pierce, Rockford, IL, USA) and quantified by the Bradford method. Total protein $(50 \mu \mathrm{g})$ was subjected to $10 \%$ SDS-PAGE and electrotransferred to PVDF membranes (Millipore, Billerica, MA, USA), which were blocked with blocking buffer (Beyotime, China) for $1 \mathrm{~h}$ at room temperature. The primary CDKN3 rabbit polyclonal antibody (1:1000; Santa Cruz Biotechnology, USA) and mouse anti- $\beta$-actin antibody (1:1000; Santa Cruz Biotechnology) were incubated on the PVDF membranes at $4^{\circ} \mathrm{C}$ overnight. The membranes were incubated with secondary antibodies for $2 \mathrm{~h}$ at room temperature (1:5000; Beyotime). The relative protein levels were visualized using an ECL system (Pierce).

Cell culture and siRNA treatment. OVCAR3 cancer cells were obtained from the Cell Biology China Academy of Science (Shanghai, China) and cultured in RPMI-1640 medium (Invitrogen, Carlsbad, CA, USA) containing 10\% fetal calf serum. The cells were cultured in a 24-well plate at a density of $6 \times 10^{4}$ cells/well and transfected with siRNA-CDKN3 or Neg. siRNA using the Lipofectamine LTX reagent (Invitrogen) according to the manufacturer's protocol. CDKN3 siRNAs (purchased from GenePharma Co., Ltd., Shanghai, China) were as follow: CCAUCAAGCAAUACAAUUATT (si-CDKN3\#1) and CUGCUUGUCUCCUACUAUATT (si-CDKN3\#2).
Table I. Correlation of CDKN3 expression and the clinicopathological characteristics of the EOC patients.

\begin{tabular}{|c|c|c|c|}
\hline \multirow[b]{2}{*}{ Characteristics } & \multicolumn{2}{|c|}{ CDKN3 expression } & \multirow[b]{2}{*}{ P-value } \\
\hline & High n (\%) & Low n (\%) & \\
\hline Total cases & $66(68.0 \%)$ & $31(32.0 \%)$ & \\
\hline Age (years) & & & 0.216 \\
\hline$\geq 58$ & 16 & 8 & \\
\hline$<58$ & 50 & 13 & \\
\hline Histological type & & & 0.879 \\
\hline Serous & $52(68.4 \%)$ & $24(31.6 \%)$ & \\
\hline Mucinous & $14(66.7 \%)$ & $7(33.3 \%)$ & \\
\hline Residual tumor size & & & 0.021 \\
\hline$<1 \mathrm{~cm}$ & $22(55.0 \%)$ & $18(45.0 \%)$ & \\
\hline$\geq 1 \mathrm{~cm}$ & $44(77.2 \%)$ & $13(22.8 \%)$ & \\
\hline FIGO stage & & & $\mathbf{0 . 0 2 0}$ \\
\hline $\mathrm{I} / \mathrm{II}$ & $20(54.1 \%)$ & $17(45.9 \%)$ & \\
\hline III/IV & $46(76.7 \%)$ & $14(23.3 \%)$ & \\
\hline Grade & & & 0.126 \\
\hline G1 & $20(57.1 \%)$ & $15(42.9 \%)$ & \\
\hline G2 & $13(65.0 \%)$ & $7(35.0 \%)$ & \\
\hline G3 & $33(78.6 \%)$ & $9(21.4 \%)$ & \\
\hline Recurrence & & & 0.017 \\
\hline Yes & $58(73.4 \%)$ & $21(26.6 \%)$ & \\
\hline No & $8(44.4 \%)$ & $10(55.6 \%)$ & \\
\hline Serum CA-125 & & & 0.199 \\
\hline$<35 \mathrm{U} / \mathrm{ml}$ & $27(61.4 \%)$ & $17(38.6 \%)$ & \\
\hline$\geq 35 \mathrm{U} / \mathrm{ml}$ & $39(73.6 \%)$ & $14(26.4 \%)$ & \\
\hline Metastasis & & & 0.610 \\
\hline Yes & $27(71.1 \%)$ & $11(28.9 \%)$ & \\
\hline No & $39(66.1 \%)$ & $20(33.9 \%)$ & \\
\hline
\end{tabular}

EOC, epithelial ovarian cancer. Bold indicates significant difference.

Colony formation and MTT assay. For the colony formation assay, 100 cells were plated into 6-well plate culture dishes and incubated for 10 days. Plates were stained with $0.1 \%$ crystal violet, and colonies with more than 50 cells were counted. For the MTT assay, cells were plated at $\sim 2000$ cells/ well in 96-well plates in medium containing $10 \%$ FBS $24 \mathrm{~h}$ after transfection. Then, $20 \mu \mathrm{l}$ of $5 \mathrm{mg} / \mathrm{ml}$ MTT (KeyGen Bio., Nanjing, China) solution was added to each well and incubated for $4 \mathrm{~h}$ at $37^{\circ} \mathrm{C}$. The media were removed from each well, and the resultant MTT formazan product was solubilized in $150 \mu \mathrm{l}$ of DMSO. The results were quantitated using a test wavelength of $490 \mathrm{~nm}$.

Cell cycle analysis. OVCAR3 cells were plated in a 6-well plate at a density of $1 \times 10^{5}$ cells/well. Cells were collected after $24 \mathrm{~h}$ and washed in cold PBS, and fixed in 75\% cold ethanol. Then the cells were centrifuged at $800 \mathrm{rpm}$ and washed with 

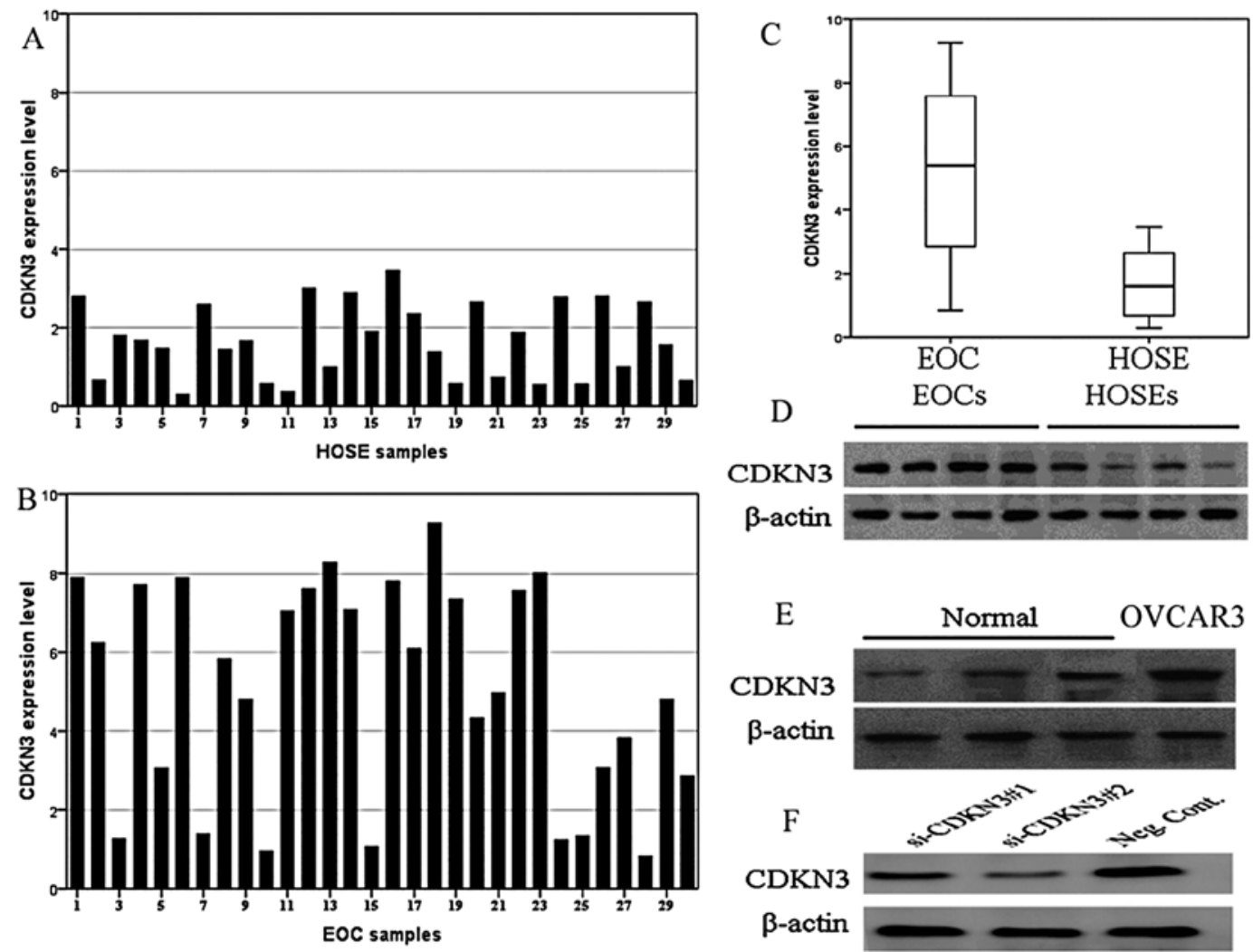

Figure 1. Expression of CDKN3 as determined by western blotting. Levels of CDKN3 expression in (A) HOSE and (B) EOC samples from 30 patients, respectively. Densitometry was normalized to $\beta$-actin. (C) Mean level of CDKN3 expression in EOC and HOSE tissues from 30 patients respectively. (D) The expression of CDKN3 protein identified by western blotting in EOC and HOSE samples. (E) The expression of CDKN3 in OVCAR3 cells compared to HOSE samples (Normal). (F) Western blot analysis of CDKN3 protein expression in OVCAR3 cells transfected with the CDKN3 siRNAs. EOC, epithelial ovarian cancer; HOSE, human ovarian surface epithelial.

cold PBS. RNase A and propidium iodide solution were added, and the cells were incubated at room temperature for $30 \mathrm{~min}$ in the dark. Cell cycle analysis was performed in a flow cytometer (FACSCalibur; BD Biosciences, USA), and ModFit LT software was used to analyze the percentage of cells in each cell cycle phase.

Transwell assay. OVCAR3 cells $\left(5 \times 10^{4}\right)$, with $200 \mu 1$ serumfree RMPI-1640, were added to the upper layer which was coated with $20 \mu 1$ Matrigel (1:4 dilution; Costar, Corning, NY, USA). RMPI-1640 containing 10\% FBS was added to the lower layer, and incubation was carried out for $24 \mathrm{~h}$ in a cell incubator. The cells that penetrated the membrane of the chamber were stained with $0.1 \%$ crystal violet for $20 \mathrm{~min}$. Cells on the upper membrane were wiped off with a cotton tip. The number of invasive cells were analyzed in 5 random fields under a microscope. The experiments were performed in triplicate.

Statistical analysis. The statistical data were analyzed by SPSS 13.0. The correlation between CDKN3 expression and EOC patient clinicopathological features was analyzed using the $\chi^{2}$ test. The t-test was used to compare messenger RNA (mRNA) in various groups. Kaplan-Meier method was used to evaluate the patient overall survival. A Cox regression model was used to demonstrate the univariate and multivariate analyses of prognostic variables. $\mathrm{P}<0.05$ was considered to indicate a statistically significant result.

\section{Results}

CDKN3 expression in the EOC tissue samples and cell lines. CDKN3 protein levels in 30 EOC and 30 HOSE tissues were determined and the results showed that $\mathrm{CDKN} 3$ was expressed weakly in the HOSE tissues (20/30), while CDKN3 expression was upregulated $>2$-fold in $76.7 \%$ (23/30) of the EOC tissues (EOC/HOSE >2) (Fig. 1A and B). The mean level of CDKN3 expression in the EOC tissues was 3.35-fold that in the HOSE tissues (Fig. 1C). Western blotting analysis revealed that expression of the CDKN3 protein was markedly higher in the EOC tissues and the OVCAR3 cell line when compared with the levels in the HOSE samples (Fig. 1D and E). In addition, CDKN3 protein expression in OVCAR3 cells transfected with the CDKN3 siRNA showed efficient depletion (Fig. 1F). As shown in Fig. 2, the IHC staining intensity and positivity of CDKN3 in the representative tissues were highly expressed $(66 / 97,68.0 \%)$ and weakly expressed $(31 / 97,32.0 \%$; Fig. 2A and $\mathrm{B})$, while negative expression was noted in the HOSE tissues (Fig. 2C).

Correlation of the clinical characteristics and CDKN3 expression in the EOC cases. CDKN3 expression levels and clinicopathological characteristics of the EOC patients are summarized in Table I. High expression of CDKN3 was detected in $68.0 \%(66 / 97)$ of the patients and low expression of CDKN3 was noted in $32.0 \%$ (31/97) of the EOC patients. 
A

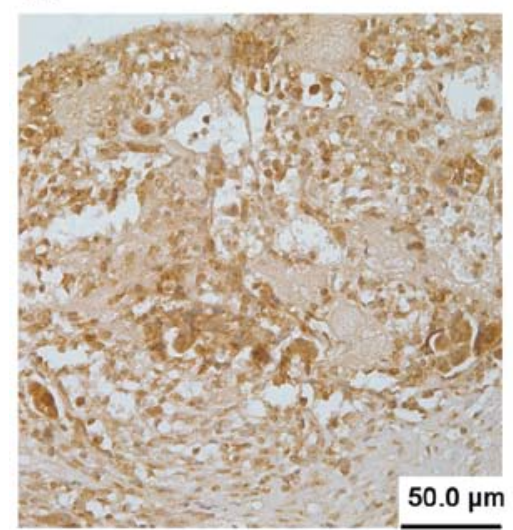

High Expression EOC
B

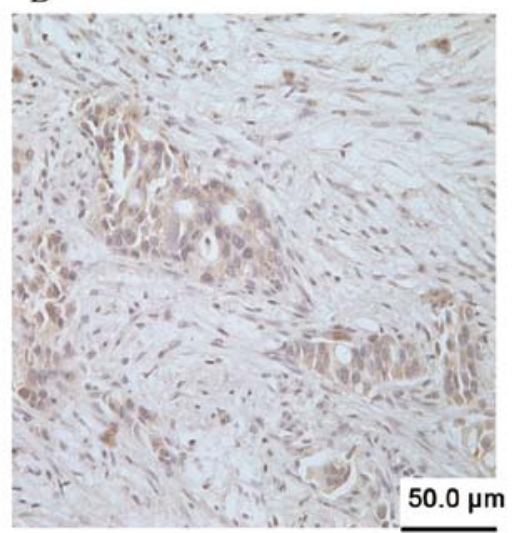

Low Expression EOC

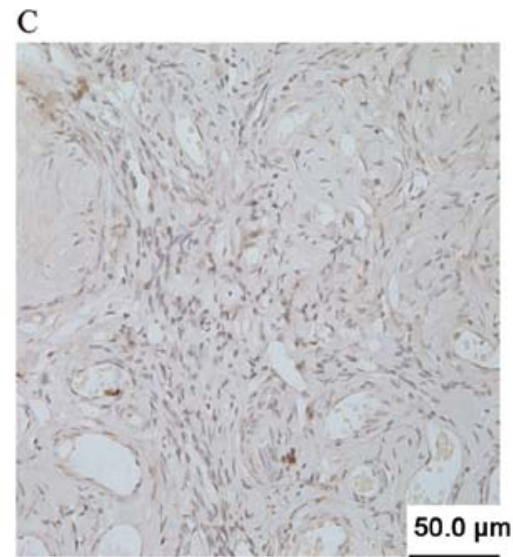

Negative Expression HOSE

Figure 2. Immunohistochemical staining of CDKN3 in the EOC and HOSE samples. (A) High expression in EOC tissue; (B) low expression in EOC tissue; (C) negative expression in HOSE tissue (immunohistochemistry, x400). EOC, epithelial ovarian cancer; HOSE, human ovarian surface epithelial.
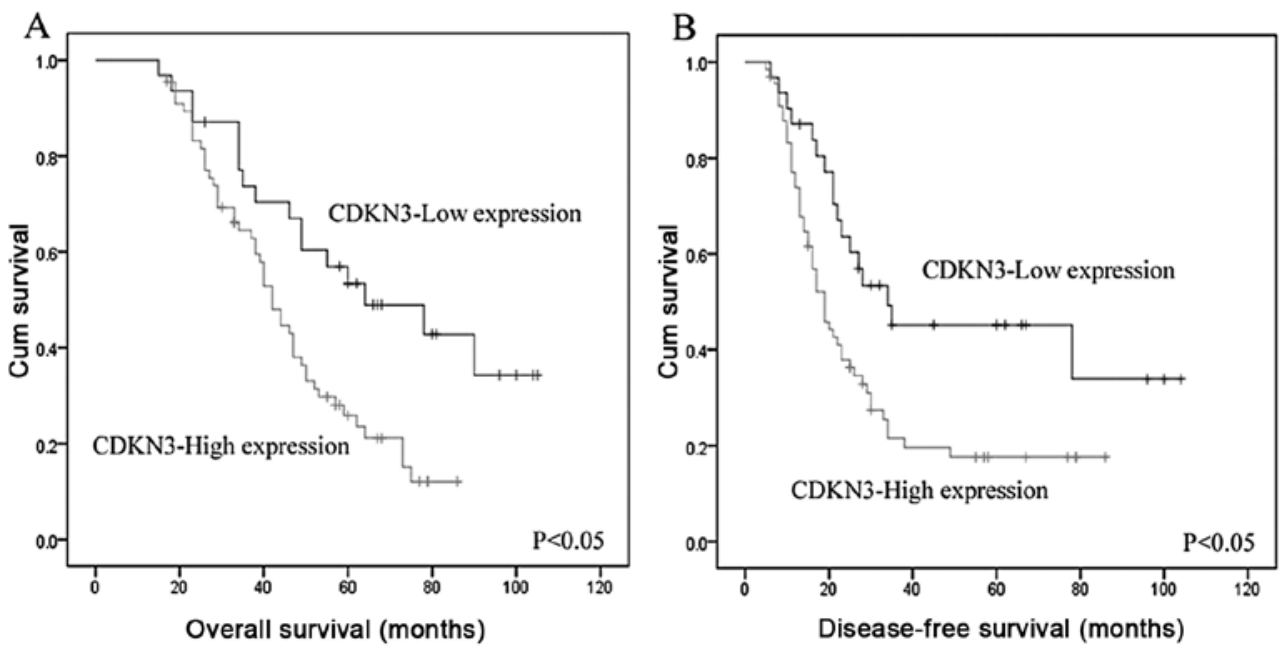

Figure 3. Kaplan-Meier analysis of the correlation between CDKN3 expression and EOC patient survival. The overall (A) and the disease-free (B) survival of EOC patients with high and low expression are shown. EOC, epithelial ovarian cancer.

High levels of CDKN3 expression were significantly associated with FIGO stage, recurrence and residual tumor size, but no significant correlation was noted with patient age, tumor type, serum Ca-125 level and histological type. Overall survival and disease-free survival were significantly reduced in patients with high CDKN3 expression than in patients with low CDKN3 expression ( $\mathrm{P}<0.05$, Fig. $3 \mathrm{~A}$ and $\mathrm{B})$. Furthermore, multivariate analysis demonstrated that a high level of CDKN3 expression was an independent predictor of prognosis of EOC patients, and was associated with increased risk of a poor prognosis (hazard ratio, 1.661; $\mathrm{P}=0.005$ ) (Table II).

Depletion of CDKN3 expression suppresses OVCAR3 cell proliferation. MTT assay showed that downregulation of CDKN3 expression significantly reduced the viability of the OVCAR3 cell line (Fig. 4A). The depletion of CDKN3 in the OVCAR3 cells (si-CDKN3\#1 and si-CDKN3\#2 vs. Neg. Cont.: $45 \pm 11$ and $34 \pm 9$ vs. $81 \pm 13 ; \mathrm{P}<0.05$ ) also led to a significant reduction in the number and size of foci (Fig. 4B). The DNA content using flow cytometry demonstrated that CDKN3 siRNA transfection increased the percentage of cells in the G1 phase and decreased those in the $\mathrm{S}$ phase in the OVCAR3 cell line ( $\mathrm{P}<0.05$, Fig. 5). As shown in Fig. 6, the depletion of CDKN3 in OVCAR3 cells did not have a measurable blocking effect on cell invasion.

\section{Discussion}

The mechanisms of the cell cycle and cancer is one of the 'hot' subjects in the field of oncology research in recent years. The cell cycle is the basic process of life activity. Under normal circumstance, cells enter into the physiological state of proliferation, differentiation, aging and death through normal cell cycle phases. If abnormal cell cycle regulation occurs, the cell enters into a cancerous state. Thus, cell cycle regulation is the core event of cell proliferation regulation, which has a close relationship with cellular carcinogenesis. Involved in cell cycle regulation are: cell cycle proteins (cyclins), cell cycle protein-dependent kinases (cyclin-dependent kinases, CDKs) and cell cycle protein kinase inhibitors (cyclin-dependent kinase inhibitors, $\mathrm{CKI} / \mathrm{CDKN}$ ). Cyclins consist of 8 members, 
Table II. Univariate and multivariate analyses of factors correlated with overall survival of the EOC patients.

\begin{tabular}{|c|c|c|c|c|c|c|}
\hline \multirow[b]{2}{*}{ Variables } & \multicolumn{3}{|c|}{ Univariate analysis } & \multicolumn{3}{|c|}{ Multivariate analysis } \\
\hline & HR & $95 \% \mathrm{CI}$ & $\mathrm{P}$-value & HR & $95 \% \mathrm{CI}$ & P-value \\
\hline CDKN3 & 3.264 & $1.781-5.983$ & $<0.001^{\mathrm{a}}$ & 1.661 & $1.324-4.351$ & $0.005^{\mathrm{a}}$ \\
\hline Age & 0.883 & $0.634-1.598$ & 0.775 & & & \\
\hline Serum Ca-125 level & 1.645 & $0.837-1.933$ & 1.259 & & & \\
\hline FIGO stage & 5.234 & $2.871-9.542$ & $<0.001^{\mathrm{a}}$ & 4.730 & $2.352-7.328$ & $0.011^{\mathrm{a}}$ \\
\hline Grade & 1.745 & $1.306-2.332$ & $0.001^{\mathrm{a}}$ & & & \\
\hline Histological type & 1.399 & $0.860-1.805$ & 0.345 & & & \\
\hline Residual tumor size & 4.511 & $2.605-7.814$ & $<0.001^{\mathrm{a}}$ & 4.231 & $2.219-6.942$ & $0.006^{\mathrm{a}}$ \\
\hline Recurrence & 3.486 & $1.504-8.082$ & $0.004^{\mathrm{a}}$ & & & \\
\hline Metastasis & 1.462 & $0.895-2.389$ & 0.129 & & & \\
\hline
\end{tabular}

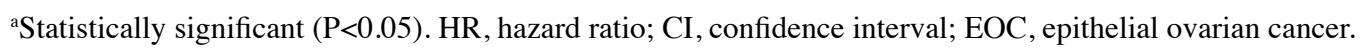

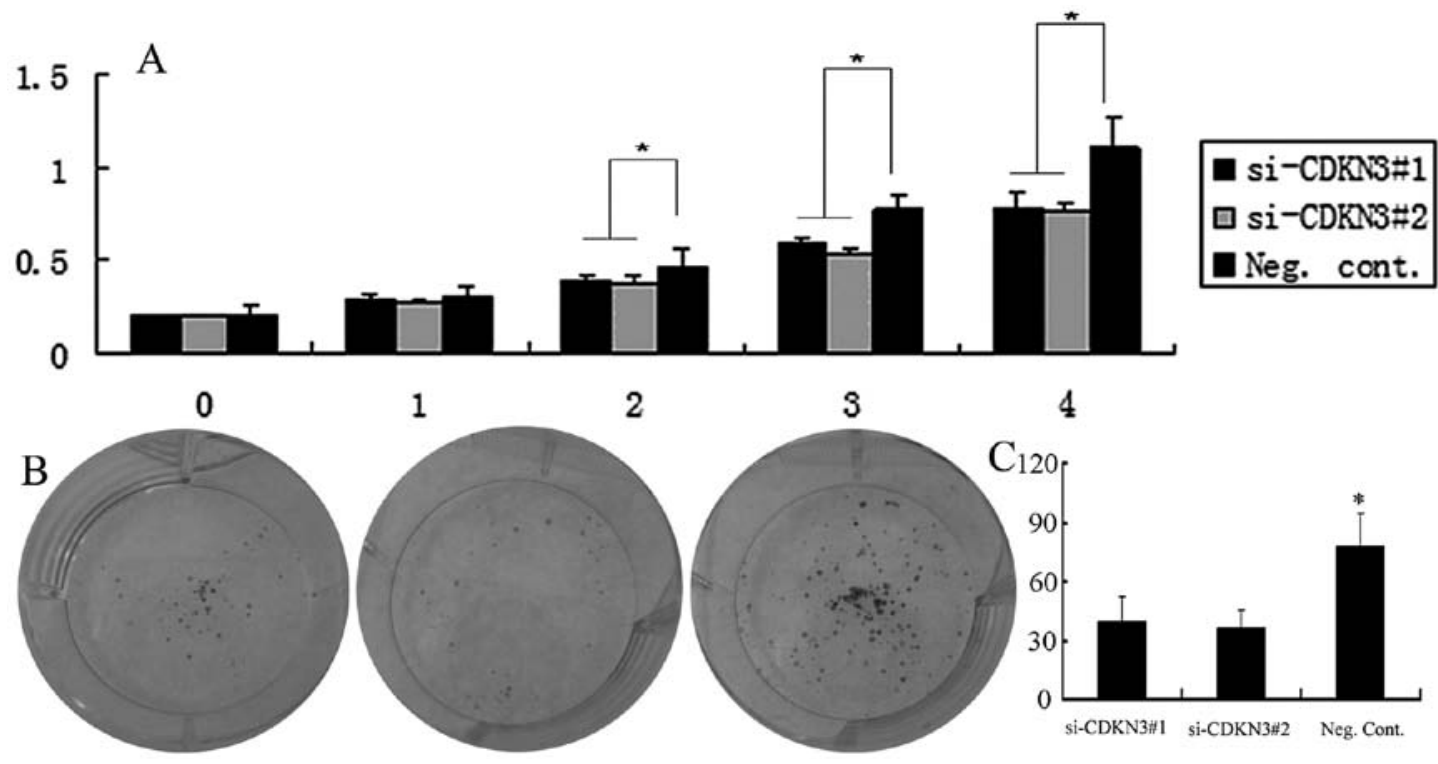

Figure 4. CDKN3 depletion impairs cancer cell proliferation. (A) MTT assay was performed following CDKN3 siRNA treatment of OVCAR3 cells. A reduction of absorbance was observed ( $\mathrm{P}<0.05$ ). (B) Assessment of the clonogenic potential of the CDKN3-depleted OVCAR3 cells. (C) The number of colonies was determined. The number of colonies formed by the OVCAR3 cells treated with CDKN3 siRNAs was far less than the number of colonies in the control cells. Each of the experiments was repeated in triplicate.

respectively named cyclin A to $\mathrm{H}$. CDK consist of 7 members, respectively named CDK1-7.

$\mathrm{CKI} / \mathrm{CDKN}$, a newly discovered protein, inhibits CDK kinase activity combined with $\mathrm{CDK}$, cyclin or cyclin-CDK complex. In the present study, 97 EOC samples were analyzed by $\chi^{2}$ test, indicating that CDKN3 expression had a close relationship with EOC proliferation and tumor recurrence, and it may play a significant role in tumor carcinogenesis and EOC progression. Based on Kaplan-Meier analysis, we discovered that high expression of CDKN3 indicates the poor prognosis of EOC patients, suggesting that it can represent a novel prognostic factor for EOC. This was the first time we evaluated the relationship among $\mathrm{CDKN} 3$, clinicopathological features and prognosis in EOC. Furthermore, we recognized that CDKN3 may be used as a novel independent prognostic biomarker to predict the overall survival rate of EOC patients based on the univariate and multivariate Cox hazards analysis. This function of $\mathrm{CDKN} 3$ corroborated the results of studies concerning renal cancers and hepatocellular carcinomas $(18,19)$. In previous research, several predictors similar to CDKN3, such as p19INK4d (20), CD163 (21), KPNA2 (22) and p21Waf1/ Cip1 (23), were recognized as being related to the prognosis of EOC. To date, however, it is still not definitely confirmed whether indices of this type could be a substitute for predicting the prognosis of EOC. Therefore, further research must be carried out to determine whether CDKN3 together with other molecules of this category may be valuable for determining the prognosis of EOC.

To better understand the biological function of CDKN3, we investigated whether depletion of CDKN3 reduces the 

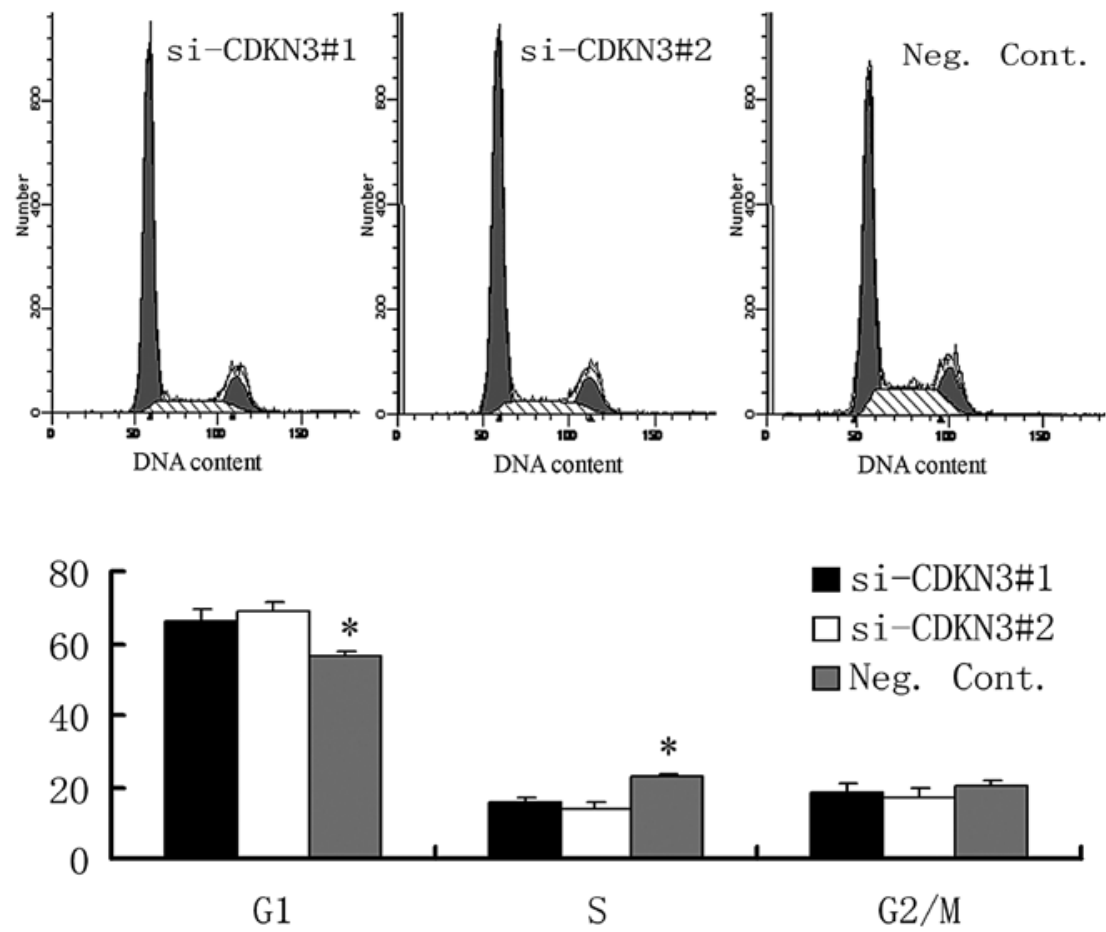

- si-CDKN3\#1

$\square$ si-CDKN3\#2

$\square$ Neg. Cont.

Figure 5. DNA content analysis revealed that siRNA-mediated CDKN3 knockdown increased the percentage of OVCAR3 cells in the G1 phase and decreased the percentage of cells in the $\mathrm{S}$ phase. The graph (bottom panel) shows the relative cell percentage in each cell cycle phase.

A

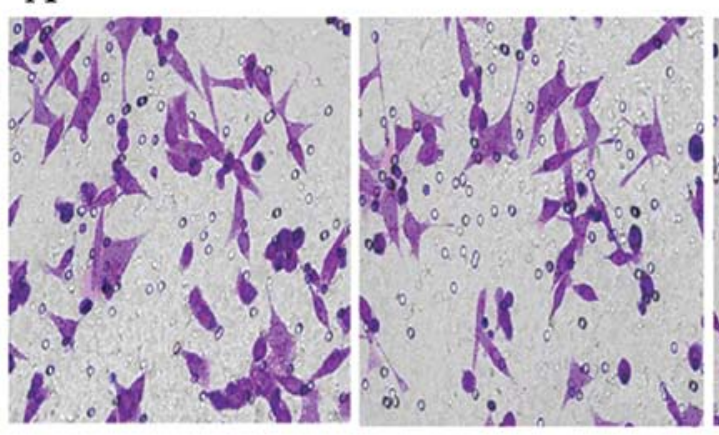

$\mathrm{B}$

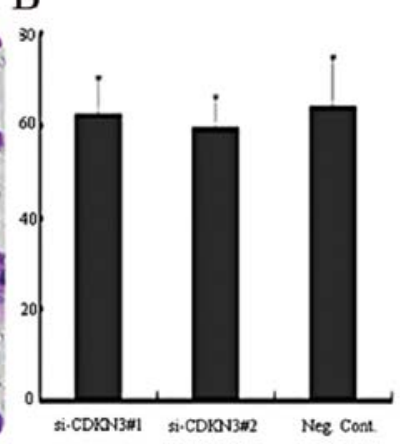

Figure 6. (A) CDKN3 siRNA treatment did not have a significant blocking effect on cell invasion in the OVCAR3 cell line. (B) The number of invasive cells was counted and no difference was observed among the cell graph.

malignant phenotypes in EOC cell lines. CDKN3 was previously found to be relatively highly expressed in various cancer cell lines $(18,23)$. Antisense KAP (CDKN3) in HeLa and LNCaP cells exhibited S-phase reduction and also suppression of the cell growth rate in vivo and in vitro (24). Accordingly, we reasoned that CDKN3 may play a role in the regulation of cell growth in ovarian cancer cell lines. We examined the colony formation potential and cell growth rate in OVCAR3 cells following siRNA treatment. We found that OVCAR3 knockdown caused a significant reduction in the proliferation rate and colony formation ability. Therefore, CDKN3 overexpression stimulated malignant cell proliferation. Moreover, we analyzed the role of CDKN3 on cell cycle progression, similar to previous studies $(16,25,26), \mathrm{CDKN} 3$ inhibited cell cycle progression in OVCAR3 cells. These data together demonstrated that CDKN3 had a measurable effect on ovarian cancer cell proliferation.
Invasion and metastasis are biological characteristics of malignant tumors, and pose the most problems for clinical treatments. Recent studies indicate that CDKN3 is positively correlated with metastasis of neuroendocrine tumors when compared with their non-metastatic counterparts (27). However, in our study, we did not find such an association. Transwell cell migration assays showed that CDKN3 knockdown did not have a measurable effect on OVCAR3 cell invasion. This finding is coincident with the clinical data of Xing et al (16) in liver cancer. A possible explanation is possibly that tumor cells which express CDKN3 are not able to migrate from the primary tumor site, leading to reduction in metastatic potential, yet CDKN3 expression could support tumor cell survival and proliferation. In addition, CDKN3 may have different influence on cancer cells in various cancer types.

Another issue involves the mechanism of how CDKN3 promotes ovarian cancer progression. A previous study 
reported that $\mathrm{p} 21$ interacts with cyclin/CDK complexes and PCNA to inhibit their kinase activities (28), and CDKN3 could promote the proliferation of liver cells through a p53-p21 manner to induce G1/S phase transition (16). Other studies have shown that CDKN3 through its protein product KAP regulates the cell cycle. It regulates retinoblastoma protein ( $\mathrm{pRb}$ ) activation via a phosphorylation mechanism that is responsible for G1 to $\mathrm{S}$ transition $(29,30)$. Currently, one hypothesis of this mechanism is the hypomethylation of its promoter region (9). This hypothesis warrants further study.

In conclusion, this study identified CDKN3 as an oncoprotein overexpressed in EOC which is important for the maintenance of the malignant phenotype and recognized CDKN3 as a candidate target protein for future cancer therapeutics.

\section{Acknowledgements}

This study was supported by grants from the National Natural Science Foundation of China (no. 81171649 to Yi Guo).

\section{References}

1. Jemal A, Siegel R, Xu J and Ward E: Cancer statistics, 2010. CA Cancer J Clin 60: 277-300, 2010.

2. Dundr P: Ovarian carcinoma: current diagnostic principles. Cesk Patol 46: 53-61, 2010 (In Czech).

3. Seidman JD, Ronnett BM and Kurman RJ: Pathology of borderline (low malignant potential) ovarian tumours. Best Pract Res Clin Obstet Gynaecol 16: 499-512, 2002.

4. Seidman JD and Kurman RJ: Pathology of ovarian carcinoma. Hematol Oncol Clin North Am 17: 909-925, vii, 2003.

5. Westfall D, Roma AA and Silva EG: High-grade serous carcinoma of the ovary. Ann Diagn Pathol 13: 285-290, 2009.

6. Vang R, Shih Ie M and Kurman RJ: Ovarian low-grade and highgrade serous carcinoma: pathogenesis, clinicopathologic and molecular biologic features, and diagnostic problems. Adv Anat Pathol 16: 267-282, 2009.

7. Tarin D: Inappropriate gene expression in human cancer and its far-reaching biological and clinical significance. Cancer Metastasis Rev 31: 21-39, 2012

8. Patterson KI, Brummer T, O'Brien PM and Daly RJ: Dualspecificity phosphatases: critical regulators with diverse cellular targets. Biochem J 418: 475-489, 2009.

9. Niculescu MD, Yamamuro Y and Zeisel SH: Choline availability modulates human neuroblastoma cell proliferation and alters the methylation of the promoter region of the cyclin-dependent kinase inhibitor 3 gene. J Neurochem 89: 1252-1259, 2004.

10. Yeh CT, Lu SC, Chen TC, Peng CY and Liaw YF: Aberrant transcripts of the cyclin-dependent kinase-associated protein phosphatase in hepatocellular carcinoma. Cancer Res 60: 4697-4700, 2000.

11. Yeh CT, Lu SC, Chao CH and Chao ML: Abolishment of the interaction between cyclin-dependent kinase 2 and Cdk-associated protein phosphatase by a truncated KAP mutant. Biochem Biophys Res Commun 305: 311-314, 2003.

12. Blum R, Nakdimon I, Goldberg L, et al: E2F1 identified by promoter and biochemical analysis as a central target of glioblastoma cell-cycle arrest in response to Ras inhibition. Int $\mathbf{J}$ Cancer 119: 527-538, 2006.
13. Wu L, Timmers C, Maiti B, et al: The E2F1-3 transcription factors are essential for cellular proliferation. Nature 414: 457-462, 2001

14. Demetrick DJ, Matsumoto S, Hannon GJ, et al: Chromosomal mapping of the genes for the human cell cycle proteins cyclin $\mathrm{C}$ (CCNC), cyclin E (CCNE), p21 (CDKN1) and KAP (CDKN3). Cytogenet Cell Genet 69: 190-192, 1995.

15. MacDermed DM, Khodarev NN, Pitroda SP, et al: MUC1associated proliferation signature predicts outcomes in lung adenocarcinoma patients. BMC Med Genomics 3: 16, 2010.

16. Xing C, Xie H, Zhou L, et al: Cyclin-dependent kinase inhibitor 3 is overexpressed in hepatocellular carcinoma and promotes tumor cell proliferation. Biochem Biophys Res Commun 420: 29-35, 2012.

17. Taylor KJ, Sims AH, Liang L, et al: Dynamic changes in gene expression in vivo predict prognosis of tamoxifen-treated patients with breast cancer. Breast Cancer Res 12: R39, 2010.

18. Lai MW, Chen TC, Pang ST and Yeh CT: Overexpression of cyclin-dependent kinase-associated protein phosphatase enhances cell proliferation in renal cancer cells. Urol Oncol 30 871-878, 2012.

19. Wang L, Sun L, Huang J and Jiang M: Cyclin-dependent kinase inhibitor $3(\mathrm{CDKN} 3)$ novel cell cycle computational network between human non-malignancy associated hepatitis/cirrhosis and hepatocellular carcinoma (HCC) transformation. Cell Prolif 44: 291-299, 2011.

20. Felisiak-Golabek A, Dansonka-Mieszkowska A, Rzepecka I, et al: p19 mRNA and protein expression as new prognostic factor in ovarian cancer patients. Cancer Biol Ther 14: 973-981, 2013.

21. No JH, Moon JM, Kim K and Kim YB: Prognostic significance of serum soluble CD163 level in patients with epithelial ovarian cancer. Gynecol Obstet Invest 75: 263-267, 2013.

22. Huang L, Wang HY, Li JD, et al: KPNA2 promotes cell proliferation and tumorigenicity in epithelial ovarian carcinoma through upregulation of c-Myc and downregulation of FOXO3a. Cell Death Dis 4: e745, 2013.

23. Bali A, O'Brien PM, Edwards LS, Sutherland RL, Hacker NF and Henshall SM: Cyclin D1, p53, and p21Waf1/Cip1 expression is predictive of poor clinical outcome in serous epithelial ovarian cancer. Clin Cancer Res 10: 5168-5177, 2004.

24. Lee SW, Reimer CL, Fang L, Iruela-Arispe ML and Aaronson SA: Overexpression of kinase-associated phosphatase (KAP) in breast and prostate cancer and inhibition of the transformed phenotype by antisense KAP expression. Mol Cell Biol 20: 1723-1732, 2000.

25. Chinami M, Yano Y, Yang X, et al: Binding of HTm4 to cyclindependent kinase (Cdk)-associated phosphatase (KAP). Cdk2. cyclin A complex enhances the phosphatase activity of KAP, dissociates cyclin A, and facilitates KAP dephosphorylation of Cdk2. J Biol Chem 280: 17235-17242, 2005.

26. Yu Y,Jiang X, Schoch BS, Carroll RS, Black PM and Johnson MD: Aberrant splicing of cyclin-dependent kinase-associated protein phosphatase KAP increases proliferation and migration in glioblastoma. Cancer Res 67: 130-138, 2007.

27. Lee J, Sung CO, Lee EJ, et al: Metastasis of neuroendocrine tumors are characterized by increased cell proliferation and reduced expression of the ATM gene. PLoS One 7: e34456, 2012.

28. Chen J, Jackson PK, Kirschner MW and Dutta A: Separate domains of p21 involved in the inhibition of Cdk kinase and PCNA. Nature 374: 386-388, 1995.

29. Barford D: The mechanism of protein kinase regulation by protein phosphatases. Biochem Soc Trans 29: 385-391, 2001.

30. Johnson LN, De Moliner E, Brown NR, et al: Structural studies with inhibitors of the cell cycle regulatory kinase cyclindependent protein kinase 2. Pharmacol Ther 93: 113-124, 2002. 